Check for updates

Cite this: Mater. Adv., 2021, 2, 4075

Received 30th December 2020, Accepted 6th May 2021

DOI: 10.1039/d0ma01026j

rsc.li/materials-advances

\title{
Formation of various-axis-oriented wurtzite nuclei and enlargement of the a-axis-oriented region in AlFeN films deposited on $\mathrm{Si}(100)$ substrates $\dagger$
}

\author{
Saki Imada, (D)*a Toshiyuki Isshiki, (D) a Nobuyuki Tatemizo, (D) $\ddagger^{a}$ Koji Nishio, (D) a \\ Shuichi Mamishin, ${ }^{\text {b }}$ Yuya Suzuki, ${ }^{\text {b }}$ Katsuji Ito, ${ }^{b}$ Kiyofumi Nitta, ${ }^{c}$ Hiroki Suga, \\ Oki Sekizawa ${ }^{c}$ and Yusuke Tamenori (D) $^{c}$
}

\begin{abstract}
Wurtzite AIN films with high Fe concentrations were deposited on Si(100) substrates using radiofrequency sputtering, and the growth process was investigated. X-Ray diffraction (XRD) analysis with parallel incident $X$-rays showed that a thick film $(\sim 2.5 \mu \mathrm{m})$ had a non-polar a-axis orientation, similar to the films deposited on $\mathrm{SiO}_{2}$ glass substrates. Through powder XRD analysis, it was found that nonwurtzite fine crystals and/or amorphous materials formed during the initial stage of deposition, followed by wurtzite nucleation with various orientations of the axes, such as $a, m$, and $r$ orientations. The XRD results also implied that the a-axis-oriented regions drastically increased with increasing film thickness. Scanning transmission electron microscopy (STEM) and energy-dispersive $\mathrm{X}$-ray spectroscopy analyses suggested that one of the candidates for the non-wurtzite fine crystalline material was $\gamma-\mathrm{Al}_{2} \mathrm{O}_{3}$. STEM analysis also demonstrated that a-axis-oriented single-crystal grains grew from randomly oriented small wurtzite grains that increased in diameter with increasing deposition time. Al K-edge X-ray absorption near-edge structure results suggested that the electronic structure in the polar $c$-axis direction of wurtzite lying in the film plane developed with deposition time, thereby further supporting the findings of the XRD and STEM analyses.
\end{abstract}

\section{Introduction}

Deep ultraviolet (DUV) sterilisation technologies have attracted increasing attention due to the COVID-19 pandemic. ${ }^{1}$ At present, DUV light sources include excimer, mercury, and metal halide lamps. DUV light-emitting diodes (LEDs) are promising options that offer the advantages of being light and small, thus leading to the realisation of handy and portable sterilisers. DUV-LEDs are rapidly being designed and developed around the world.

Wurtzite aluminium nitride (AlN) is a direct and wide bandgap $(\sim 6.2 \mathrm{eV}, 200 \mathrm{~nm})^{2}$ semiconductor that is a prospective material for the fabrication of DUV-LEDs. AlN belongs to the family of III-nitrides centred on GaN, which are successfully used for fabricating visible-light LEDs. Thus, constructing DUV-LEDs

\footnotetext{
${ }^{a}$ Faculty of Electrical Engineering and Electronics, Kyoto Institute of Technology, Kyoto, 606-8585, Japan. E-mail: saki_imada@kit.ac.jp; Tel: +81-75-724-7484

${ }^{b}$ Hitachi High-Tech Corporation, 1040, Ichige, Hitachinaka, Ibaraki, 312-8504, Japan

${ }^{c}$ Japan Synchrotron Radiation Research Institute, Sayo, Hyogo, 679-5198, Japan $\dagger$ Electronic supplementary information (ESI) available. See DOI: 10.1039/ d0ma01026j

\# Present address: Nippon Steel Corporation, Japan.
}

with AlN is straightforward. However, its practical application is not easy: the $c$-axis ([0002]) orientation of films, which is the easy orientation among III-nitrides prepared using conventional techniques based on epitaxial growth regimes, is a major impediment, particularly for AlN. For example, the large difference in electronegativity between $\mathrm{Al}$ and $\mathrm{N}$ causes a quantum-confined Stark effect (QCSE) in vertical quantum-well LED structures. ${ }^{3,4}$ The QCSE leads to a low recombination probability of electrons and holes, resulting in low emission efficiency. In addition to the QCSE problem, the split regimes at the top of the valence band (VB) of wurtzite AlN can cause another problem: dipole transitions between the top of the $\mathrm{VB}$ and the bottom of the conduction band (CB) are forbidden for light with electric fields in the $c$-plane, which results in low extraction efficiency in vertical LED structures. ${ }^{5}$

As these problems are traced back to the polar $c$-axis orientation of thin films, aligning the $c$-axis in film planes is the fundamental solution. Thus, intensive efforts have been invested in growing AlN films with non-polar oriented axes ([11-20], $a$-axis, and $[10-10], m$-axis $)^{6-8}$ and semi-polar ones $([10-13]$ and $[11-22]) .{ }^{9}$ However, the problem encountered in the early phase of GaN film development still persists, namely, the lack of suitable substrates for growing such AlN films. ${ }^{10,11}$ 
We have been studying the effects of the partial substitution of $\mathrm{Al}$ in AlN films with a 3d transition metal on the crystallographic properties and band structures of these films. ${ }^{12-16} \mathrm{In}$ a previous study, we found that AlN films containing Fe (AlFeN) on $\mathrm{SiO}_{2}$ glass substrates prepared with radio-frequency (RF) sputtering exhibited an $a$-axis orientation when the Fe concentration exceeded $10 \% .{ }^{15}$ Furthermore, thermal annealing of the films can eliminate Fe from the lattices while maintaining the $a$-axis orientation..$^{16}$ Thus, we propose heavy Fe substitution and post-annealing as a new technique to obtain a seed layer to prepare non-polar AlN films. ${ }^{16}$

In this study, $\mathrm{Si}(100)$ was selected as the substrate because the difference in the thermal expansion coefficient between $\mathrm{Si}$ and $\mathrm{AlN}$ is smaller than that between $\mathrm{SiO}_{2}$ and AlN. Moreover, $\mathrm{Si}$ is a substrate not only for LEDs ${ }^{17}$ but also for other electronic devices. ${ }^{18}$ Therefore, non-polar AlN films formed on $\mathrm{Si}(100)$ substrates are considered excellent materials for producing high-efficiency devices.

Sputtering is a successful commercial method to obtain thin films such as metal alloys and compounds, ${ }^{19}$ including $c$-axisoriented AlN films. ${ }^{20}$ Recently, some studies have reported that non-polar $m$-axis-oriented AlN films can be obtained via the optimisation of growth conditions such as the $\mathrm{Ar} / \mathrm{N}_{2}$ gas ratio and total gas pressure during sputtering. ${ }^{21-23}$ These films were deposited on $\mathrm{SiO}_{2}$ glass substrates via spontaneous nucleation, which is one of the characteristics of sputtering. Recently, sputtering was used to obtain non-polar $m$-axis-oriented AlN films on a $\mathrm{Si}(100)$ substrate with a MnS buffer layer ${ }^{24}$ and nonpolar $a$-axis-oriented AlN films on an $r$-plane sapphire substrate,$^{25}$ both of which exhibited epitaxial growth regimes. The successful fabrication of these films proves the potential of the sputtering technique for promoting epitaxial growth to construct devices comprising non-polar AlN-based layers. One of our research aims is to fabricate a seed layer for such devices using the all-sputtering technique.

\section{Materials and methods}

AlFeN films were deposited on $\mathrm{Si}(100)$ substrates using $\mathrm{RF}$ sputtering (ULVAC KIKO, Inc., RFS-200S) from an AlN target (Furuuchi Chemical Corp., 99\%, $80 \mathrm{~mm}$ in diameter) with $\mathrm{Fe}$ metal chips (Furuuchi Chemical Corp., 99.9\%, $5 \mathrm{~mm}$ square). The surface area ratio of the target (Fe:AlN) was $\sim 1: 7$ for all samples. The distance between the sputter target and the substrate was $55 \mathrm{~mm}$. The base vacuum condition achieved before the deposition was $7 \times 10^{-4} \mathrm{~Pa}$. The RF power was $200 \mathrm{~W}$. The deposition temperature was $300{ }^{\circ} \mathrm{C}$, and an $\mathrm{Ar} / \mathrm{N}_{2}$ gaseous mixture $(2: 1)$ at a total pressure of $0.65 \mathrm{~Pa}$ was used (both with $6 \mathrm{~N}$ purity procured from Iwatani Gas). Except for the substrate, the deposition conditions were the same as those used for $a$-axis-oriented AlFeN films $(\sim 19 \% \mathrm{Fe})$ in our previous study ${ }^{15}$ Preprocessing to remove the native oxidised layer on the Si substrate was not carried out. Neither an adhesion layer nor a buffer layer was used. Before starting the deposition, the target was cleaned (i.e., pre-sputtering) for $20 \mathrm{~min}$. The deposition
Table 1 Thicknesses and Fe concentrations estimated by XRF analysis

\begin{tabular}{lll}
\hline & \multicolumn{2}{l}{ Estimated values by XRF } \\
\cline { 2 - 3 } Deposition time $(\mathrm{h})$ & Thickness $(\mu \mathrm{m})$ & Fe concentration $(\%)$ \\
\hline 5.0 & 2.5 & 18.6 \\
2.5 & 0.9 & 15.1 \\
1.0 & 0.4 & 14.0
\end{tabular}

times were 1.0, 2.5, and $5.0 \mathrm{~h}$ (referred to herein as $1.0 \mathrm{~h}-, 2.5 \mathrm{~h}$-, and $5.0 \mathrm{~h}$-films, respectively). The Fe concentrations and thicknesses of the films were estimated using X-ray fluorescence (XRF) analysis (Hitachi High-Tech Corp., SEA5120), as summarised in Table 1. The thickness and Fe concentration of the $5.0 \mathrm{~h}$-film are comparable to those of the films on the $\mathrm{SiO}_{2}$ glass substrates with an $a$-axis orientation..$^{15,16}$ The sample structure model used to estimate the film thicknesses and $\mathrm{Fe}$ concentrations through XRF analysis was an AlFeN layer on a $\mathrm{Si}$ substrate. As discussed later, this model is not strictly appropriate for evaluating films formed using a short deposition time (1.0 h-film), indicating that the volume of the interfacial layers between AlFeN and the Si substrate should be considered.

X-Ray diffraction (XRD) measurements were carried out using two apparatuses. One was designed for thin-film samples, equipped with a two-crystal $(\mathrm{Ge}(220))$ monochromator that makes incident X-rays parallel. This apparatus was the same as that used in our previous investigations (PANalytical, X'Pert MRD). ${ }^{15,16}$ The other was designed for powder samples without a monochromator (Rigaku, MiniFlex 600). The former (TF-XRD) detects diffractions from planes parallel to the substrate, whereas the latter (P-XRD) can detect diffractions from planes of slightly tilted small crystals due to intense incident X-rays passing through the $\mathrm{Cu} \mathrm{K} \beta$ filter and collimator. Both measurements were conducted in the $\theta / 2 \theta$ mode with $\mathrm{Cu} \mathrm{K} \alpha$ radiation. The XRD powder diffraction profiles were simulated using VESTA. ${ }^{26}$

Cross-sectional scanning transmission electron microscopy (STEM) images and energy-dispersive X-ray (EDX) element depth profiles were obtained using an HD-2700 system (Hitachi High-Tech Corp.) equipped with a silicon drift detector (SDD; Oxford, X-manN 100 TLE). Samples were prepared using a focused ion beam system (Hitachi High-Tech Corp., NB5000) with a Ga ion beam.

Al K-edge X-ray absorption near-edge structure (XANES) measurements were performed at BL27SU of SPring-8 in the total electron yield mode under vacuum $\left(\sim 10^{-6} \mathrm{~Pa}\right)$ at room temperature. The electric field vectors $(E)$ of the incident X-rays were parallel to the film plane. Calculations of the $\mathrm{Al} \mathrm{K}$-edge XANES spectra of wurtzite AlN were performed using the FDMNES code. ${ }^{27,28}$ The lattice constants obtained from the XRD measurements for the $5.0 \mathrm{~h}$-film $(a=3.166 \AA$ and $c=5.050 \AA)$ were applied to the model. The internal parameter, $u$, was 0.378 . The cluster radius used for the calculation was $6.6 \AA$ (110 atoms were included). After the self-consistent field calculation, the $\mathrm{Al}$ K-edge XANES spectra were calculated under two different conditions, namely, muffin-tin approximation with multiple scattering theory (MT-MST) and full potential (FP) without MST. $^{29}$ 


\section{Results and discussion}

Fig. 1(a) shows the XRD profiles of the samples obtained using TF-XRD. The $5.0 \mathrm{~h}$-film exhibits a strong peak at $58^{\circ}$ and a considerably smaller peak at $33^{\circ}$, corresponding to wurtzite AlN $(11-20)$ and $(10-10)$, respectively; the peaks are shifted to slightly smaller angles because of an increase in the lattice constant caused by Fe substituting Al. These results indicate that the preferred orientation of the $5.0 \mathrm{~h}$-film on $\mathrm{Si}(100)$ is along the $a$-axis, similar to that of the films on $\mathrm{SiO}_{2}$ glass. ${ }^{15,16}$ For the $2.5 \mathrm{~h}$-film, although the intensity of the peak at $33^{\circ}$ is comparable to that of the $5.0 \mathrm{~h}$-film, the peak at $58^{\circ}$ is significantly smaller. Both these peaks are exceedingly small for the $1.0 \mathrm{~h}$-film. In Fig. 1(b), the deposition time dependences of the $(11-20)$ and $(10-10)$ peak intensities divided by the deposition times are plotted. It was implied that $a$-axis-oriented regions developed as deposition progressed, whereas $m$-axisoriented regions formed only during the initial stage of deposition.

Fig. 1(c) shows the P-XRD and simulated powder diffraction profiles for the same samples. The model used for the simulation was a wurtzite AlN structure with lattice constants $a=3.166 \AA$ and $c=5.050 \AA$; the simulated profile coincided well with the P-XRD profile of the $5.0 \mathrm{~h}$-film with these lattice constants. Through $\mathrm{P}$-XRD, peaks at $37^{\circ}$ were detected, which correspond to wurtzite AlN (10-11). In addition, wide halo structures between $15^{\circ}$ and $35^{\circ}$ appeared in the P-XRD profiles. A halo structure was observed for the Si substrate; however, the intensity was low, presumably due to a native oxide layer. An increase in the halo intensity indicates that a fine crystalline layer and/or an amorphous layer were formed during the deposition of AlFeN. Because there is no plane of wurtzite AlN (AlFeN) with lattice spacing $d$ between $15^{\circ}$ and $35^{\circ}$, the halo structure did not originate from wurtzite AlN. (a)

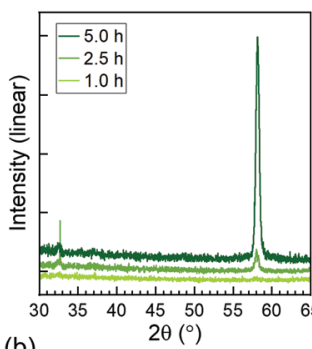

(b)

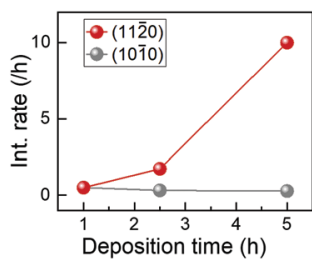

(c)

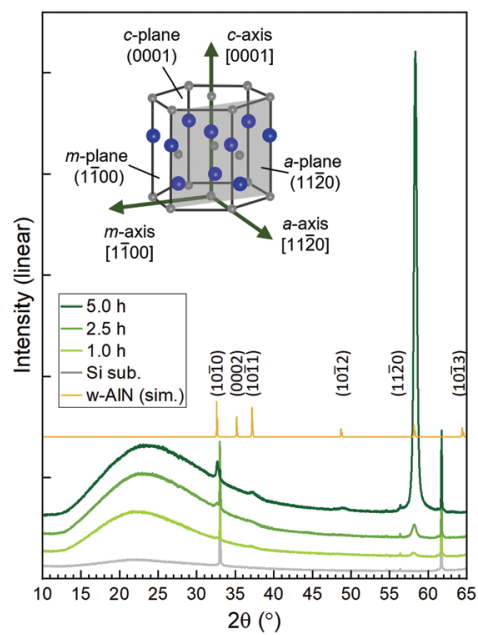

Fig. 1 (a) XRD profiles of the samples obtained using TF-XRD. (b) Deposition time dependences of (11-20) and (10-10) peak intensities divided by deposition times. (c) XRD profiles of the samples in (a) formed on Si(100) substrates obtained using P-XRD and a simulated powder diffraction profile of wurtzite AIN. Profiles in panels (a) and (c) are shifted in the direction of the longitudinal axis.
STEM analysis was carried out for the $5.0 \mathrm{~h}$-film. The brightfield (BF) STEM image of the overall cross-section is shown in Fig. 2(a). The film consists of roughly three regions: a thin region located directly above the Si substrate with clear interfaces, a region with an indistinct grain-boundary configuration, and a columnar crystal region, indicated as A, B, and $\mathrm{C}$, respectively. Fig. 2(b) shows a magnified image obtained from the vicinity of the interface between the substrate and the film. The red and yellow arrows serve as rough guides indicating the thicknesses of the $2.5 \mathrm{~h}$ - and $1.0 \mathrm{~h}$-films, respectively. In conjunction with the deposition time dependence of the P-XRD profiles, regions A and B are believed to be the source of the halo structure.

Representative Fourier-transform (FT) patterns corresponding to the STEM images of regions B and A were obtained and are shown in Fig. 2(c) and (d), respectively. Some grains crystallised in region $\mathrm{B}$, producing ring-like $\mathrm{FT}$ patterns that implied randomly oriented wurtzite. The positions of (10-10), (0002), (10-11), (10-12), and (11-20) are indicated by red circular arcs in Fig. 2(c) from the centre to the edge. A bright region is observed within the pattern of $(10-10)$; this finding agrees with the halo observed in the P-XRD profiles. Conversely, in region A (Fig. 2(d)), small crystals are recognisable as spotty FT patterns. The positions of the spots differ from those in region $\mathrm{B}$. The most distinct pattern is the innermost one. Because neither wurtzite nor rock-salt AlN contains planes in this region (see Fig. S1, ESI $\dagger$ ), materials other than AlN are responsible for the FT patterns of region A. To identify the material, we performed EDX analysis. The results are depicted as depth profiles in Fig. 2(e) over the corresponding region of the STEM image in Fig. 2(f). The results indicate that region A consists of $\mathrm{Al}, \mathrm{O}$, and $\mathrm{Fe}$. As oxygen gas was not supplied during the deposition, oxygen could have originated from the native oxide layer of the Si substrate, residual oxidation layers of the AlN target and Fe metal chips, and residual impurities in the growth chamber. At present, it is not clear whether region A consists of an $\mathrm{Al}-\mathrm{Fe}$ oxide or a mixture of $\mathrm{Al}$ and $\mathrm{Fe}$ oxides; however, the FT patterns in Fig. 2(d) can be explained based on the (111), (220), (311), (400), (511), and (440) planes of $\gamma-\mathrm{Al}_{2} \mathrm{O}_{3}$ from the centre to the edge (blue quarters of circles).

In region $\mathrm{B}$, the nitrogen content increased with decreasing oxygen content. In agreement with this, larger crystals grew, as observed in Fig. 2(f). Fig. 2(g)-1-(g)-4 show the FT patterns of the areas indicated by squares in Fig. 2(f). Fig. 2(g)-1 (corresponding to square 1 ) is in the region where oxygen signals were only weakly observed, and the corresponding FT pattern indicates randomly oriented wurtzite. The black column, which indicates a single crystalline structure in the BF-STEM image, started from the point in square 2. Its FT pattern shows spots in the growth direction, as indicated by red triangles, which correspond to (11-20). In Fig. 2(g)-3, the spots corresponding to $(10-10)$ are indicated by green triangles. The intensities of the spots associated with $(11-20)$ and $(10-10)$ increase with further deposition, owing to the enlargement of the singlecrystal region (i.e., the occupancy of the single-crystal region in the areas increases). Fig. 2(h) shows the FT patterns corresponding 

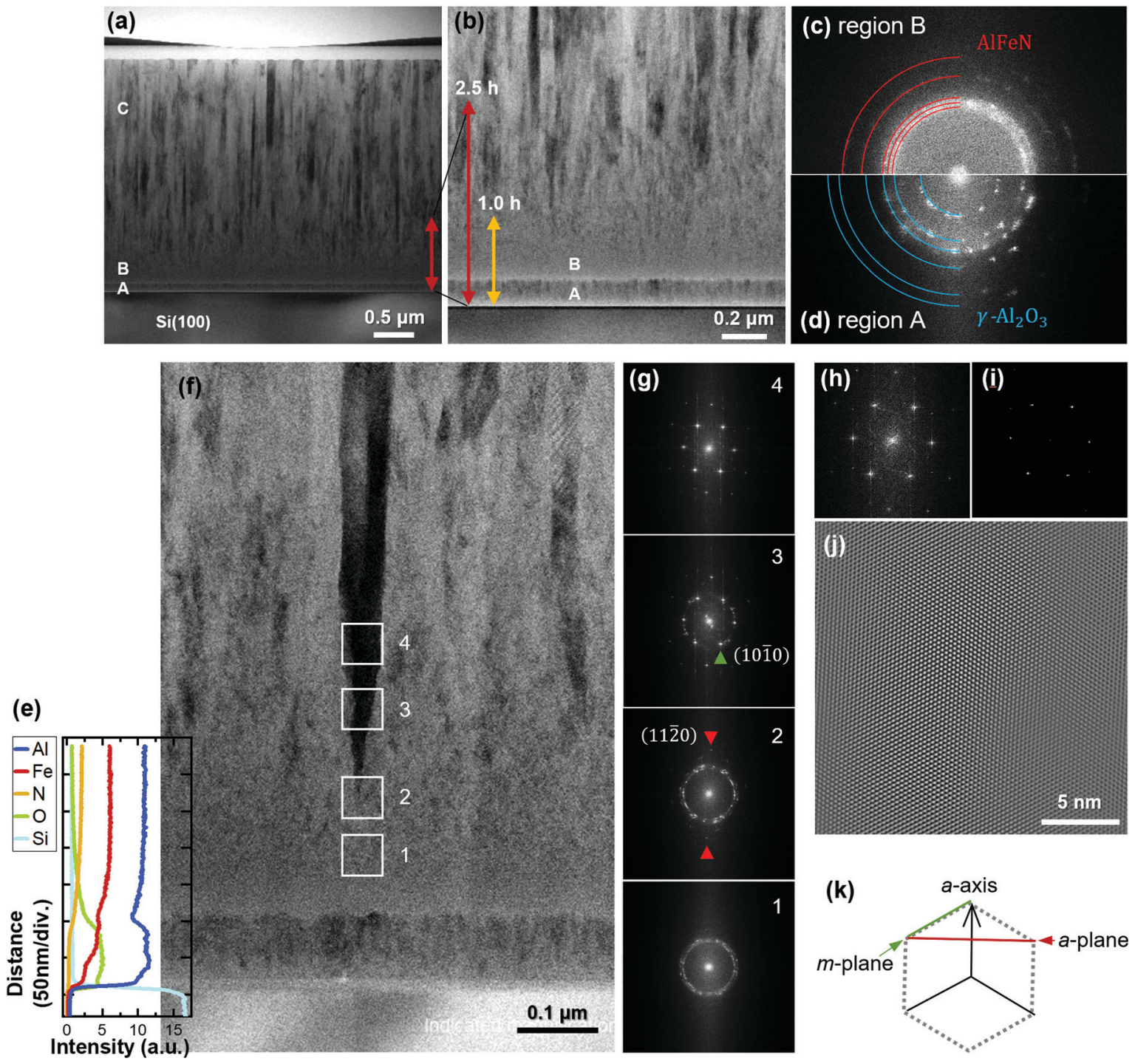

Fig. 2 STEM images of the $5.0 \mathrm{~h}$-film: (a) overall cross-section, (b) interface between the substrate and film. Red and yellow arrows are rough guides indicating the thicknesses of the $2.5 \mathrm{~h}$ - and $1.0 \mathrm{~h}$-films, respectively. Fourier-transform (FT) patterns corresponding to (c) region B and (d) region A. (e) EDX profiles. Intensities of the profiles are proportional to the $\mathrm{K} \alpha$ line intensities of $\mathrm{Al}, \mathrm{Fe}, \mathrm{N}$, O, and Si. (f) STEM image with a representative singlecrystal pillar. (g) Corresponding FT patterns of the areas enclosed by squares in (f). (h) FT pattern of the centre in (f)-4. (i) Extracted strong spots from (h). (j) Inverse FT image of (i) and (k) the corresponding schematic diagram showing planes.

to the centre of square 4, and Fig. 2(i) shows the strong patterns extracted from Fig. 2(h). The inverse FT image shown in Fig. 2(i) confirms the $a$-axis orientation of wurtzite, as shown in Fig. 2(j) with (k).

If $a$-axis-oriented single-crystal grains can be selected from various-axis-oriented wurtzite nuclei and become enlarged with increasing deposition times, then the electronic structures are expected to change accordingly. To investigate the electronic structures of the films, Al K-edge XANES measurements were carried out in the total electron yield mode, which is a surfacesensitive technique (a few nm in depth). Because the electric field vector $(E)$ of the incident X-ray was parallel to the film plane, the spectra reflected the in-plane electronic structure of the film. Fig. 3(a) depicts the spectra of $1.0 \mathrm{~h}-, 2.5 \mathrm{~h}$-, and $5.0 \mathrm{~h}$-films normalised at energies higher than $1579 \mathrm{eV}$. The spectra consist of four main peaks $\left(\mathrm{M}_{1}-\mathrm{M}_{4}\right)$ with a small pre-edge peak $(\mathrm{P})$, which are homologous with the spectra of the $a$-axis-oriented AlFeN films on $\mathrm{SiO}_{2}$ substrates. ${ }^{15,16}$ Each peak showed a different deposition time dependence in the normalised spectra; the intensity of $\mathbf{M}_{1}$ did not change, whereas that of $\mathbf{M}_{3}$ decreased with the deposition time. $\mathbf{M}_{2}$ and $\mathbf{M}_{4}$ became more pronounced. In addition, the intensity of $\mathrm{P}$ increased slightly. In our previous study, it was found that the intensities of pre-edge peaks did not depend on $E$ of the incident X-rays, as supported by theoretical band calculations. ${ }^{15,16}$ Thus, the intensity change of $\mathrm{P}$ was attributed to the increase in the Fe concentration.

The theoretical spectra of wurtzite AlN for the $E / / a$-axis, $E / / c$-axis, and powder (no specific orientation) are shown in Fig. 3(b). Peaks $\mathrm{M}_{\mathrm{t} 2}$ and $\mathrm{M}_{\mathrm{t} 4}$ represent the features of the $E / / c$-axis and peak $\mathrm{M}_{\mathrm{t} 3}$ corresponds to the $E / / a$-axis; peak $\mathrm{M}_{\mathrm{t} 1}$ is present in 

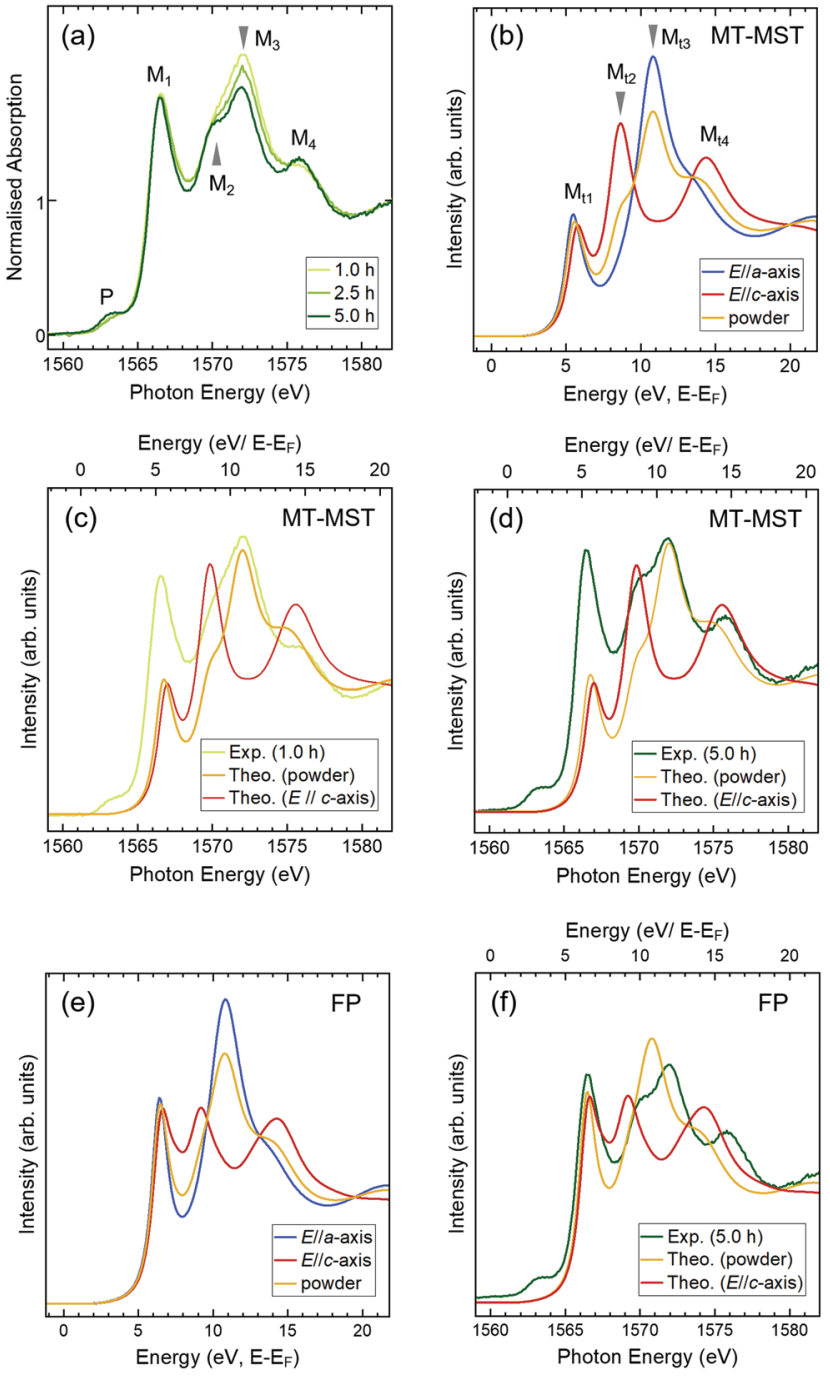

Fig. 3 (a) Deposition time dependence of Al K-edge XANES spectra. (b) Theoretical spectra of w-AIN for the E//a-axis, E//c-axis, and W-AIN powder obtained using MT-MST. Comparison of the theoretical powder and E//C-axis spectra with the experimental spectra of (c) $1.0 \mathrm{~h}$ - and (d) $5.0 \mathrm{~h}$-films. (e) Theoretical spectra of $\mathrm{w}$-AIN for the E//a-axis, E//c-axis, and w-AIN powder calculated with FP and (f) comparison with the experimental spectrum of the $5.0 \mathrm{~h}$-film.

both cases. In the spectrum of the powder, these features are averaged. The spectrum of the $1.0 \mathrm{~h}$-film resembles that of the powder model shown in Fig. 3(c), which is consistent with the STEM analysis results, implying that the $1.0 \mathrm{~h}$-film consists of randomly oriented wurtzite crystals at the surface (region B). As for the $5.0 \mathrm{~h}$-film, it is clear that the spectrum contains strong features of the $E / / c$-axis (Fig. 3(d)) (because the film had no specific preferred orientation in the film plane, the spectrum of the $5.0 \mathrm{~h}$-film contains some features of the powder spectrum.) These findings suggest that the polar $c$-axis lies in the film plane of the $5.0 \mathrm{~h}$-film. These XANES results agree with those of the STEM and XRD analyses. It is interesting to note that the intensities of $\mathbf{M}_{1}$ are reproducible when performing FP calculations (Fig. 3(e) and (f)). As $\mathbf{M}_{\mathrm{t} 1}$ reflects the shape at the very bottom of the CB in AlN, this consistently sharp and strong peak implies that the CBs of the AlFeN films are well-organised, even at high Fe concentrations.

\section{Conclusions}

Wurtzite AlN films with high Fe concentrations were deposited on $\mathrm{Si}(100)$ substrates via RF sputtering and investigated by performing XRD, STEM, and Al K-edge XANES analyses. The XRD analysis showed that the thick films $(\sim 2.5 \mu \mathrm{m})$ displayed non-polar $a$-axis orientation of wurtzite, similar to the films on $\mathrm{SiO}_{2}$ glass substrates reported in our previous studies. ${ }^{15,16}$ These findings suggest that $a$-axis-oriented films can be obtained independent of the substrate, thereby implying the self-organisation of $a$-axis-oriented nuclei or selective growth of an $a$-axis-oriented region from an initially various-axis oriented region. The deposition time dependence of the films analysed using XRD and XANES in conjunction with STEM suggests that the latter is the probable mechanism.

In this study, it was found that $\gamma-\mathrm{Al}_{2} \mathrm{O}_{3}$-type fine crystals were formed during the initial stage of deposition. As oxygen gas was not supplied during the deposition, oxygen is believed to have originated from the native oxide layer of the Si substrate, residual impurities in the growth chamber, and residual oxidation layers of the AlN target and Fe metal chips. This oxide layer can be a barrier for using a Si substrate as the back-surface contact for an LED. Presumably, the surface of a metal target can be cleaned more easily than that of a nitride target. Thus, $\mathrm{Al}$ and Fe metal targets may be more suitable to avoid the formation of such an oxide layer.

The role of $\mathrm{Fe}$ in the enlargement of the $a$-axis-oriented single crystals remains unclear. It is possible that either (i) the Fe atoms act as surfactants or (ii) the incorporation of Fe atoms in the AlN lattice is essential to form bonds, resulting in $a$-axis orientation. It is worth noting that AlTiN, ${ }^{13} \mathrm{AlVN},{ }^{14}$ and $\mathrm{AlCrN}^{12}$ films do not indicate any orientation change from the $c$ - to the $a$-axis at the transition metal concentrations exceeding $10 \%$, whereas the AlFeN films began to exhibit a gradual orientation change at an Fe concentration of approximately $5 \% \cdot{ }^{15,16} \mathrm{We}$ will conduct comparative studies using other $3 \mathrm{~d}$ transition metals including $\mathrm{Co}$ and $\mathrm{Ni}$ for elucidating the role of $\mathrm{Fe}$ in $a$-axis-oriented growth, which is indispensable for obtaining large and highly crystalline seed layers to realise efficient DUV-LEDs.

\section{Conflicts of interest}

There are no conflicts to declare.

\section{Acknowledgements}

The authors would like to thank Prof. T. Araki of Ritsumeikan University for permission to use the XRD apparatus (TF-XRD) in his laboratory. Al K-edge XANES measurements were performed at the BL27SU of SPring-8 with the approval of the JASRI (No. 2020A1257). This work was partly supported by JSPS KAKENHI 
(No. 20K04562) and the Iketani Science and Technology Foundation (No. 0311021-A).

\section{References}

1 M. Buonanno, D. Welch, I. Shuryak and D. J. Brenner, Sci. Rep., 2020, 10, 1-8.

2 I. Vurgaftman, J. R. Meyer and L. R. Ram-Mohan, J. Appl. Phys., 2001, 89, 5815-5875.

3 P. Waltereit, O. Brandt, A. Trampert, H. T. Grahn, J. Menniger, M. Ramsteiner, M. Reiche and K. H. Ploog, Nature, 2000, 406, 865-868.

4 K. Balakrishnan, T. Katona and A. Khan, Nat. Photonics, 2008, 2, 77-84.

5 K. Kojima, A. A. Yamaguchi, M. Funato, Y. Kawakami and S. Noda, J. Appl. Phys., 2011, 110, 043115.

6 T. Takeuchi, S. Sota, M. Katsuragawa, M. Komori, H. Takeuchi, H. Amano and I. Akasaki, Jpn. J. Appl. Phys., 1997, 36, L382-L385.

7 D. V. Dinh, N. Hu, Y. Honda, H. Amano and M. Pristovsek, J. Mater. Chem. C, 2020, 8, 8668-8675.

8 Y. Taniyasu and M. Kasu, Appl. Phys. Lett., 2010, 96, 221110.

9 D. V. Dinh, N. Hu, Y. Honda, H. Amano and M. Pristovsek, Sci. Rep., 2019, 9, 15802.

10 S. Strite, J. Vac. Sci. Technol., B: Microelectron. Nanometer Struct., 1992, 10, 1237.

11 H. Morkoç, Nitride Semiconductors and Devices, Springer, Berlin, Heidelberg, 2013.

12 N. Tatemizo, S. Imada, Y. Miura, K. Nishio and T. Isshiki, AIP Adv., 2017, 7, 055306.

13 N. Tatemizo, Y. Miura, K. Nishio, S. Hirata, F. Sawa, K. Fukui, T. Isshiki and S. Imada, J. Mater. Chem. A, 2017, 5, 20824-20832.

14 N. Tatemizo, S. Imada, Y. Miura, K. Nishio and T. Isshiki, J. Appl. Phys., 2018, 123, 161546.
15 N. Tatemizo, S. Imada, K. Nishio and T. Isshiki, AIP Adv., 2018, 8, 115117.

16 N. Tatemizo, S. Imada, K. Okahara, H. Nishikawa, K. Tsuruta, T. Ina, Y. Miura, K. Nishio and T. Isshiki, Sci. Rep., 2020, 10, 1819.

17 B. T. Tran, N. Maeda, M. Jo, D. Inoue, T. Kikitsu and H. Hirayama, Sci. Rep., 2016, 6, 3-8.

18 M. Clement, L. Vergara, J. Sangrador, E. Iborra and A. SanzHervás, Ultrasonics, 2004, 42, 403-407.

19 S. Swann, Phys. Technol., 1988, 19, 67-75.

20 G. F. Iriarte, J. G. Rodríguez and F. Calle, Mater. Res. Bull., 2010, 45, 1039-1045.

21 M. Ishihara, S. J. Li, H. Yumoto, K. Akashi and Y. Ide, Thin Solid Films, 1998, 316, 152-157.

22 M. Clement, E. Iborra, J. Sangrador, A. Sanz-Hervás, L. Vergara and M. Aguilar, J. Appl. Phys., 2003, 94, 1495-1500.

23 F. Medjani, R. Sanjinés, G. Allidi and A. Karimi, Thin Solid Films, 2006, 515, 260-265.

24 K. Tatejima, T. Nagata, K. Ishibashi, K. Takahashi, S. Suzuki, A. Ogura and T. Chikyow, Jpn. J. Appl. Phys., 2019, 58, SBBK03.

25 K. Tatejima, T. Nagata, K. Ishibashi, K. Takahashi, S. Suzuki, A. Ogura and T. Chikyow, Jpn. J. Appl. Phys., 2019, 58, SDDG07.

26 K. Momma and F. Izumi, J. Appl. Crystallogr., 2011, 44, 1272-1276.

27 O. Bunău and Y. Joly, J. Phys.: Condens. Matter, 2009, 21, 345501.

28 S. A. Guda, A. A. Guda, M. A. Soldatov, K. A. Lomachenko, A. L. Bugaev, C. Lamberti, W. Gawelda, C. Bressler, G. Smolentsev, A. V. Soldatov and Y. Joly, J. Chem. Theory Comput., 2015, 11, 4512-4521.

29 Y. Joly and S. Grenier, in X-Ray Absorption and X-Ray Emission Spectroscopy, ed. J. A. van Bokhoven and C. Lamberti, John Wiley \& Sons, Ltd, Chichester, UK, 2016, pp. 73-97. 